\title{
Evaluation of Interest-region Detectors and Descriptors for Automatic Landmark Tracking on Asteroids*
}

\author{
Naoya Takeishi, ${ }^{1)}$ Akira Tanimoto, ${ }^{1)}$ Takehisa Yairi,${ }^{2)}$ Yuichi Tsuda,${ }^{3)}$ Fuyuto Terui, ${ }^{3)}$ \\ Naoko Ogawa, ${ }^{3)}$ and Yuya Mimasu ${ }^{3)}$ \\ ${ }^{1)}$ Department of Aeronautics and Astronautics, The University of Tokyo, Tokyo 113-8656, Japan \\ ${ }^{2)}$ Research Center for Advanced Science and Technology, The University of Tokyo, Tokyo 153-8904, Japan \\ ${ }^{3)}$ Japan Aerospace Exploration Agency, Kanagawa 252-5210, Japan
}

\begin{abstract}
The asteroid explorer Hayabusa-2, which is scheduled to be launched in 2014, is going to perform a global mapping mission after it arrives at the target asteroid. Although most of the global mapping sequence will be the same as that of its predecessor Hayabusa, several automation technologies are planned to be tested to reduce the workload of the operators. In particular, the structure from motion (SFM) and simultaneous localization and mapping (SLAM) techniques are expected to significantly contribute to the automation of asteroid shape estimation and visual spacecraft navigation. These frameworks require automatic landmark tracking on the asteroid surface, but no previous work has discussed the method that should be used to track images of the asteroid taken in space, where the absence of scattering light causes dramatic changes in appearance. In this study, we evaluated the performances of SIFT, SURF, BRISK, ORB, Harris-Affine, Hessian-Affine and MSER for images of the asteroid. We found that SIFT is acceptable for use, while SURF, BRISK and ORB can be used with careful parameter tuning. The affine-invariant detectors might contribute to more accurate tracking, but using them is more challenging owing to an extra normalizing process.
\end{abstract}

Key Words: Asteroid Exploration, Interest Region Detector, Local Descriptor, Landmark Tracking, Global Mapping

\section{Introduction}

The Hayabusa spacecraft (originally called MUSES-C) managed two touchdowns at the Muses Sea of Asteroid 25143 (Itokawa) in November 2005. ${ }^{1)}$ In the Hayabusa mission, the geometric shape and motion condition of the asteroid were estimated to choose a suitable touchdown site and for navigation of the spacecraft during its descent, as well as for scientific analysis. This estimation, called global mapping, was carried out using the asteroid's limb profile and the surface shading and stereo vision techniques, ${ }^{2-4)}$ which required many manual procedures.

In Hayabusa's descending phase before it released the target marker, it was navigated by Doppler measurement and using images of the asteroid taken with an optical navigation camera (ONC). ${ }^{5)}$ The lateral position of the spacecraft was estimated by matching the landmarks in ONC images and those in the geometric model; this procedure is called ground control point navigation (GCP-NAV). In GCP-NAV for the Hayabusa mission, 70 landmarks on the asteroid surface were configured and manually tracked in the ONC images by the ground operator, which required considerable time and labor.

The successor asteroid explorer, Hayabusa-2, ${ }^{6}$ ) is scheduled to be launched in 2014. Although most of its global mapping sequence will be the same as that of Hayabusa, several automation technologies are planned to be tested to

(C) 2015 The Japan Society for Aeronautical and Space Sciences *Received 14 March 2014; final revision received 4 July 2014; accepted for publication 28 July 2014. reduce the workload of the operators. One of them is based on state-of-the-art computer vision and robotics technologies that can automate the global mapping and visual navigation. This method depends on the automatic extraction and tracking of features (landmarks) from asteroid images. Recently, several methods have been proposed for tracking landmarks automatically, and it is necessary to choose an appropriate method for successful global mapping and visual navigation. Hence, the purpose of this study is to evaluate the performance of automatic landmark-tracking techniques for global mapping and visual navigation.

\section{Related Work}

\subsection{SFM and SLAM}

In the area of computer vision, the reconstruction of threedimensional geometry from two-dimensional images has been intensively studied. This problem is called structure from motion (SFM) and usually deals with the simultaneous reconstruction of an object's geometric shape and camera positions from moving points in corresponding images. ${ }^{7)}$ There have been two major solutions for SFM: SVD-based factorization $^{8)}$ and nonlinear optimization. ${ }^{9)}$ SVD-based factorization is fast but vulnerable to noises and missing measurements, whereas nonlinear optimization is generally more accurate but slow and does not converge to the optimum solution without an appropriate initial solution.

A similar problem has been intensively studied in the area of robotics, which is called simultaneous localization and mapping (SLAM) and is used to estimate the location of a robot and a map of the environment simultaneously. The 
Table 1. Overview of interest region detectors and descriptors.

\begin{tabular}{|c|c|c|c|c|c|}
\hline & Method & Invariance & Detection & Description & Efficiency \\
\hline \multirow{4}{*}{ Cat.1 } & SIFT & \multirow{4}{*}{$\begin{array}{l}\text { Scale \& } \\
\text { Rotation }\end{array}$} & \multirow{2}{*}{ Blob } & \multirow{2}{*}{ Vector } & Medium \\
\hline & SURF & & & & High \\
\hline & BRISK & & \multirow{2}{*}{ Corner } & \multirow{2}{*}{ Binary code } & Very high \\
\hline & ORB & & & & Very high \\
\hline \multirow{3}{*}{ Cat.2 } & Harris-Affine & \multirow{3}{*}{ Affine } & Corner & \multirow{3}{*}{ - } & Medium \\
\hline & Hessian-Affine & & \multirow{2}{*}{ Blob } & & Medium \\
\hline & MSER & & & & Medium \\
\hline
\end{tabular}

SLAM problem has traditionally been solved with filtering such as Kalman filtering and particle filtering. ${ }^{10)}$

Global mapping and visual navigation can be treated using SFM or SLAM; if we define and track some landmarks on the surface of the asteroid, they can be used as moving points in SFM or the environment to be estimated in SLAM, with the spacecraft itself as the camera or the robot.

It has been previously shown that SFM or SLAM can be used for global mapping and visual navigation. Cocaud and Kubota applied Rao-Blackwellized particle filter (RBPF) SLAM to the simulation of autonomous descent navigation. ${ }^{11)}$ Mori et al. confirmed and discussed the suitability of the SFM framework for reconstructing the shape of Itokawa $^{12)}$ using the free SFM implementation named Bundler. ${ }^{13)}$ Tanimoto et al. reconstructed the shape of a mock asteroid ${ }^{14)}$ using co-embedding, ${ }^{15)}$ which is much faster than nonlinear optimization or filtering and robust to missing measurements. Takeishi discussed the suitability of automatic landmark configuration on the asteroid surface. ${ }^{16)}$ Despite these intensive studies, the best way to define and track landmarks on asteroid surfaces has never been discussed.

\subsection{Interest-region detectors and local descriptors}

One way of defining and tracking landmarks on the asteroid surface automatically is to utilize image feature detectors and local photometric descriptors. The detectors extract salient points or regions from images and the descriptors describe their photometric characteristics so that the regions can be identified and tracked in multiple images.

Many interest-region detectors and local descriptors have been proposed so far. In Table 1, we provide an overview of some of these detectors and descriptors, which are divided into two categories; scale- and rotation-invariant (Category 1) and affine-invariant (Category 2). The following subsections describe brief explorations of them. Moreover, we show examples of the interest regions detected from asteroid images in Fig. 1.

\subsubsection{Scale- and rotation-invariant techniques}

We first introduce some widely used image feature techniques with scale and rotation invariance; that is, features are invariant to scale changes and in-plane rotation of images.

\subsubsection{Real vector descriptors}

The scale-invariant feature transform (SIFT) proposed by Lowe ${ }^{17)}$ is one of the most widely used local descriptors for many applications. First, interest points in multiple scales are extracted by finding extreme values in the difference of

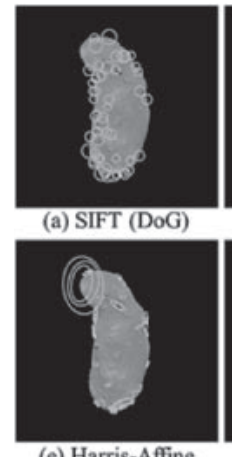

(e) Harris-Affine

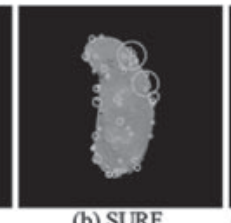

(b) SURF

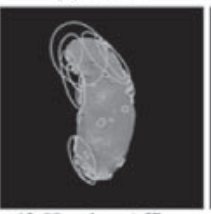

(f) Hessian-Affine

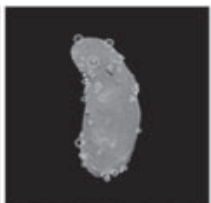

(c) BRISK (AGAST)

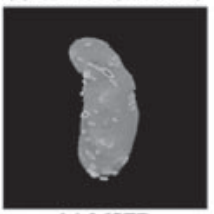

(g) MSER

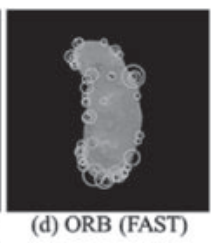

Fig. 1. Examples of interest regions detected with (a) SIFT (DoG), (b) SURF, (c) BRISK (AGAST), (d) ORB (FAST), (e) Harris-Affine, (f) Hessian-Affine and (g) MSER.

Only portions of the regions are displayed to avoid overprinting the images. The regions marked with circles or ellipses are the measurement regions on which photometric characteristics are described.

Gaussian (DoG), which is the difference between images smoothed by Gaussian convolution with multiple scales. After finding the interest points, histograms of the intensity gradient are computed in $4 \times 4$ grids around each interest point, which are quantized into eight directions. Then the dominant orientation of each interest point is estimated from the peak of the gradient histograms. The SIFT descriptor consists of these histograms normalized by the dominant orientation, thus it is a $4 \times 4 \times 8=128$-dimensional vector. The corresponding points can be tracked by calculating the similarity of the 128-dimensional descriptors in multiple images, even after a scale change or image rotation, because SIFT extracts interest points in multiple scales and estimates the dominant orientation.

Bay et al. proposed speeded-up robust features (SURF). ${ }^{18)}$ SURF utilizes box filters as an approximation of a Hessian matrix to select the locations of interest points in scale space, draws a $4 \times 4$ grid around each interest point similarly to that in SIFT and calculates the Haar wavelets in the $x$ and $y$ directions in the grids, rather than intensity gradients. The SURF descriptor consists of the sum of Haar wavelets and their absolute values in each grid, thus it is a $4 \times 4 \times 4=64$-dimensional vector. SURF detectors and descriptors are very fast compared to SIFT because of the approximation of the Hessian matrix by the box filters.

\subsubsection{Binary code descriptors}

We introduce binary robust independent elementary features (BRIEF) proposed by Calonder et al., ${ }^{19)}$ for completeness of the discussion. BRIEF is the simplest method of generating binary codes as a descriptor, and have neither scale nor rotation invariance. The BRIEF descriptor investigates the intensity differences between certain pairs of pixels around each interest point and generates binary codes according to the sign of the differences, where pairs are chosen randomly from a Gaussian distribution. BRIEF is very efficient because the extraction of intensity differences is very fast and the matching of binary-coded descriptors can be performed quickly using Hamming distances. As the interestpoint detector to be used with BRIEF, the authors suggested 
the use of FAST, proposed by Rosten and Drummond. ${ }^{20)}$ FAST is a fast and light corner detection algorithm, but it does not have any invariance itself.

Leutenegger et al. proposed binary robust invariant scalable keypoints (BRISK), ${ }^{21)}$ which is a binary code descriptor with scale and rotation invariance. The suggested detector is the AGAST corner detector, ${ }^{22)}$ which is an improved version of FAST, with investigation in a scale-space pyramid for scale invariance. The BRISK descriptor generates binary codes by examining the intensity differences between pixel pairs that are scattered around each interest point, and the dominant orientation is estimated by calculating the intensity gradient.

Rublee et al. proposed oriented FAST and rotated BRIEF $(\mathrm{ORB}){ }^{23)}$ which generate binary code descriptors similarly to BRIEF and BRISK with scale and rotation invariance. The authors suggested the use of scaled FAST as the detector, and ORB achieves rotation invariance by calculating the orientation of interest points using the difference between the intensity centroid and the geometric centroid. ORB uses a machine learning algorithm to choose pairs of pixels to maximize the variance of the descriptors and to minimize the correlation between the descriptors.

\subsubsection{Affine invariant techniques}

There is another type of invariance; that is, affine invariance. Harris affine and Hessian affine detectors were proposed by Mikolajczyk and Schmid. ${ }^{24)}$ They are based on the second-moment matrix computed around each interest point, and the interest points are searched for in affine Gaussian scale-space.

Maximally stable extremal regions (MSER) ${ }^{25)}$ were proposed by Matas et al. and consist of dark or bright extremal regions where the intensity is lower or higher than that of all the pixels on the outer boundary. Such regions are selected with a suitably optimized threshold, thus they become stable. MSERs are invariant to affine transformations as well as monotonic changes in intensity.

\subsubsection{Performance evaluation}

There have been many performance evaluations of these detectors and descriptors, including those by the proposers of the detectors or descriptors ${ }^{17-25)}$ as well as intensive comparison studies. ${ }^{26-29)}$ Although the proposers highlight the advantages of their own detector or descriptor, Heinly et al. conducted a comparison ${ }^{29)}$ of the Harris detector, ${ }^{30)}$ SIFT, SURF, BRIEF, BRISK, ORB and MSER with the result that SIFT was the best for various datasets, while binary features such as BRISK and ORB were viable with a trade-off in performance. Fraundorfer and Bischof ${ }^{28)}$ evaluated the performance of MSER, DoG, Harris-Affine, Hessian-Affine, intensity-based regions (IBR), ${ }^{31)}$ the Harris detector ${ }^{30)}$ and the Hessian detector ${ }^{30)}$ for images of nonplanar scenes. They obtained a good matching score for MSER, although the number of detected regions was relatively small compared with that for other detectors.

\footnotetext{
${ }^{1}$ http://opencv.org/

${ }^{2}$ http://www.robots.ox.ac.uk/ $v g g /$ research/affine/
}

\section{Performance Evaluation for Asteroid Images}

The evaluation papers of interest-region detectors and local descriptors ${ }^{26-29)}$ are helpful to some extent when choosing the detector and descriptor for global mapping of the asteroid or visual spacecraft navigation. However, it is notable that the photometric conditions differ more in space than on the ground, while the existing performance evaluations consider images taken only on the ground. Since asteroid-specific detectors and descriptors have not yet been developed, one of the detectors and descriptors widely used in other applications should be chosen. Previous experimental work ${ }^{11,12,14,16)}$ on global mapping or visual navigation utilized SIFT or SURF without any discussion of the detector or descriptor that should be used. Therefore, in this paper, we focus on the previously unreported area of the performance evaluation for images of an asteroid taken in space.

One of the major differences in the photometric conditions between space and the ground is that the scattering of light is not a major factor in space, resulting in a dramatic variation in the appearance of the asteroid surface. Another difference is in the opposition surge; in space, the brightness of a celestial surface may increase when the surface is illuminated directly behind an observer. This is due to the regolith of the surface and the airlessness, and cannot be observed on the earth. Moreover, the scene of the asteroid is nonplanar and its large curvature causes affine transformations of the appearance. Given such characteristics, there is no guarantee that detectors and descriptors will perform as well in space as on the ground, although it is preferable that they should work in the same manner as described in many previous studies, even for images of the asteroid.

In this study, we investigate the basic performances of widely used detectors and descriptors, SIFT, ${ }^{17)}$ SURF, ${ }^{18)}$ BRISK, ${ }^{21)} \mathrm{ORB},{ }^{23)}$ the Harris-Affine detector, ${ }^{24)}$ the Hessian-Affine detector ${ }^{24)}$ and the MSER detector, ${ }^{25)}$ for images of the asteroid. First four techniques are scale- and rotationinvariant (Category 1) and the latter three are said to have an affine-invariance (Category 2).

The purpose of this study is to investigate whether they can be used with images of the asteroid and to discuss their suitability for use in future asteroid exploration missions. SIFT achieved good results in the previously mentioned evaluation $^{29)}$ and in many applications ${ }^{7)}$; thus, we expect SIFT to also work well with images of the asteroid. We are also interested in the performances of binary descriptors such as BRISK and ORB, which are very computationally efficient and should be computable using an on-board device. Moreover, the three affine-invariant detectors might outperform the scale- and rotation-invariant detectors because the transformation of the surface appearance has strong affinity.

In the following experiments, SIFT, SURF, BRISK and ORB were computed with OpenCV ${ }^{1}$, which is one of the most well-known implementations of computer vision techniques. For the other detectors, the implementation by the Visual Geometry Group, University of Oxford, ${ }^{2}$ was used. As these implementations were not fully optimized for our 
purpose, we do not focus on the execution time or memory efficiency to ensure a fair comparison. Moreover, the parameters of the detectors and descriptors were set to the default values in the implementations in this evaluation. This setting of default parameters does not reduce the validity of our discussion because our aim is to determine the overall performance, rather than the best one of the seven methods evaluated here.

\section{Evaluation Framework}

We follow the evaluation framework proposed by Mikolajczyk et al., ${ }^{26,27)}$ with some modifications including the heuristics to obtain the ground truth. In the following, note that 'interest regions' denote the measurement regions where each descriptor is calculated, while some detectors extract only points rather than regions and others extract regions.

\subsection{Ground truth}

For a quantitative evaluation, the ground truth of region correspondences is necessary. Mikolajczyk et al. ${ }^{26)}$ used the homography between a pair of images, although the homography is not appropriate for expressing a change in viewpoint due to asteroid rotation. Fraundorfer and Bischof ${ }^{28)}$ proposed a method of giving the ground truth to nonplanar scenes using trifocal tensors, which required dense matching between images. However, this method is difficult to apply here owing to changes in the appearance of the asteroid surface.

Instead, we utilize a geometric model of an asteroid to give the ground truth of the asteroid rotation and region correspondences as respectively shown in Figs. 2 and 3. There are several asteroids with reconstructed geometric shapes, and we use the model of Asteroid 25143 (Itokawa) developed by Gaskell et al., ${ }^{4)}$ which is one of the asteroids most intensively investigated ever. First we reproject the Gaskell model, eliminating hidden vertices with z-buffering. Then we draw the boundary by finding the alpha hull of the reprojected points, and optimize the rotation parameters so as to minimize the difference between the rims in the actual image and those in reprojected image. The optimization is performed heuristically; we initially manually align the rotation parameters with the first image that captures the widest area of the asteroid, and we adjust the parameters of the other images automatically utilizing the fact that the images were taken contiguously. Then the region correspondences can be regarded as the correspondences of the aligned vertices of the Gaskell model. This concept is explained in section 4.2.4 in detail.

\subsection{Evaluation criteria}

We conduct the evaluation with five criteria: region density, region uniformity, region repeatability, ${ }^{26)}$ matching recall and matching precision. ${ }^{27)}$ Figure 4 summarizes these five criteria, which we explain in detail in the following.

\subsubsection{Region density}

For accurate reconstruction of the asteroid shape, it is desirable for the interest regions to be extracted as densely as possible. Thus, we compute the density of the detected re-

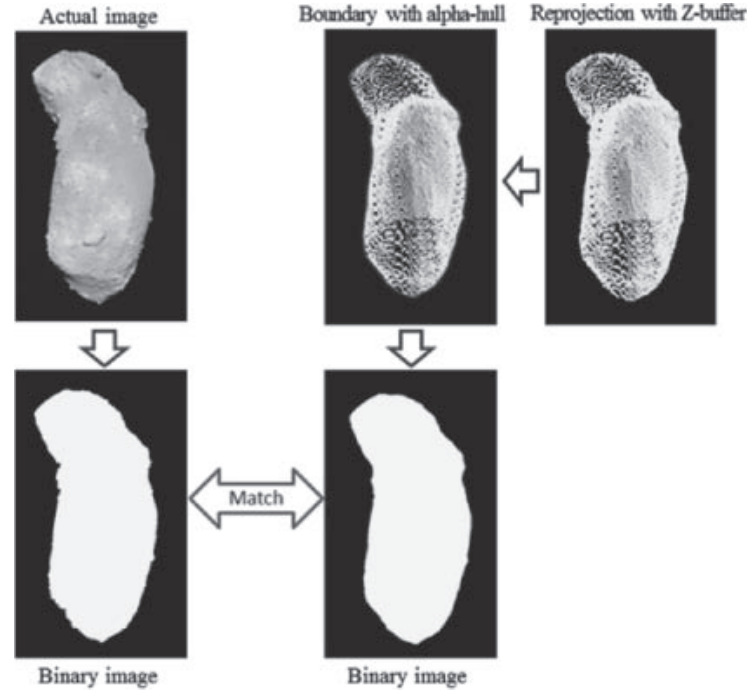

Fig. 2. Concept of estimating asteroid rotation with reprojection of the Gaskell model.

The rims of the actual asteroid and the reprojected points are matched so as to minimize their difference.

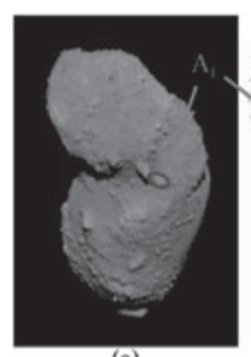

(a)

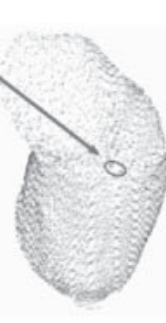

(b)

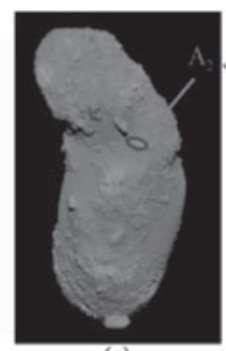

(c)

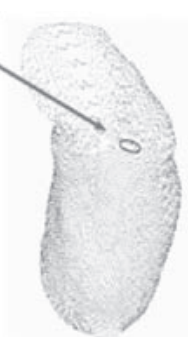

(d)
Fig. 3. Concept of finding corresponding regions using reprojection of the Gaskell model.

(a) Image with an interest region and (b) its correspondent reprojection. (c) Another image and (d) its correspondent reprojection. The overlap error between $A_{1}$ and $A_{2}$ is calculated using the intersection of the Gaskell model vertices assigned to $A_{1}$ and $A_{2}$.

gions as $D=n_{\text {inast. }} / A_{\text {inast. }}$, where $n_{\text {inast. }}$ [regions] is the number of detected regions within the asteroid and $A_{\text {inast. }}$ [px] is the area covered by the asteroid in the image.

The number of regions changes with the parameters of the detector, while detectors often extract interest regions at the same location with different scales. It is difficult to fully eliminate the redundant regions by parameter tuning for all the images; thus, we maintain the parameters at the default values, as justified in Section 3.

\subsubsection{Region uniformity}

It is desirable for the interest regions to be uniformly scattered because the shape reconstruction and visual navigation would fail in areas where few regions were extracted. Thus, we investigate the uniformity of the detected regions using a measure similar to entropy, $U=\sum_{i . j}^{H, W} \log \left(W_{i, j}\right)$, where $W_{i, j}$ is the total number of possible states of interest regions scattered in image sub-area $(i, j)$. We divide the image (originally $1,024 \times 1,024[\mathrm{px}])$ into areas of size $32 \times 32$ [px]. For example, the sub-area $(2,1)$ corresponds to the area whose coordinate is $33 \leq x \leq 64$ and $1 \leq y \leq 32$ of the orig- 
inal image. If the interest regions are distributed uniformly in each sub-area, $U$ (uniformity or entropy) becomes large. To eliminate the effect of the number of detected regions, it is normalized to about 1,000 before calculating the uniformity score.

\subsubsection{Region repeatability}

Even when the interest regions are extracted in one image, they are not always repeated in another image, although humans can easily rediscover the corresponding region, as illustrated in Fig. 4(b) with solid white (repeated) and dotted (not repeated) circles. Hence, we investigate how consistently the detector locates interest regions and give a score for repeatability of $R p=n_{\text {crp. }} / n_{\text {poscrp. }}$. Here, $n_{\text {crp. }}$ is the number of correspondences between a pair of images and $n_{\text {poscrp. }}$ is the number of possible correspondences (i.e., the number of detected regions for which more than $50 \%$ of the corresponding model vertices are contained in the reference image). The correspondences are found by investigating the overlap error described below.

\subsubsection{Overlap error}

The overlap error is defined as the proportion of the area of intersection between a pair of regions. If the overlap error is sufficiently small, the pair of regions can be regarded as corresponding. To calculate the overlap error, we assign a set of Gaskell model vertices to each interest region as shown in Fig. 3. Denoting the areas of two interest regions as $A_{1}$ in Figs. 3(a) and (b) and $A_{2}$ in Figs. 3(c) and (d), the area of intersection between the two regions is $A_{\text {int. }}=n_{\text {int. }} / d_{\text {int. }}$, where $d_{\text {int. }}$ is the density of vertices in the intersection and $n_{\text {int. }}$ is the number of Gaskell model vertices appearing in both $A_{1}$ and $A_{2} . d_{\text {int. }}$ is approximated by the mean of the densities of the two interest regions. The union area of the two regions is expressed as $A_{\text {uni. }}=A_{1}+A_{2}-A_{\text {int. }}$ and the overlap error is $e=1-A_{\text {int. }} / A_{\text {uni. }}$. A small overlap error means that the two regions are detected at very similar locations with almost the same orientation. A large overlap error denotes a displacement and change in orientation between the two regions. A pair of interest regions whose overlap error is less than $50 \%$ is regarded as corresponding in this evaluation.

To compensate for the effects of region size, we normalize the long radius of an ellipse to 20 , maintaining the ratio between the radii and the angle of the ellipse for all detectors when computing the overlap errors. ${ }^{26)}$

\subsubsection{Matching recall and precision}

The landmark matches searched for using the similarity of the descriptors do not always contain all corresponding regions and often contain false matches, as depicted in Fig. 4(b) with solid (correct) and dotted (false) lines. The ratio of correct matches to correspondences (possible matches) is used as the recall score, and the ratio of correct matches to all matches is used as the precision score. The recall score is $R c=n_{\text {corr.match }} / n_{\text {crp. }}$, where $n_{\text {corr.match }}$ is the number of correct matches, and the precision score is $\operatorname{Pr}=n_{\text {corr.match }} /$ $\left(n_{\text {corr.match }}+n_{\text {falsematch }}\right)$. Matches with overlap errors of less than $50 \%$ are regarded as correct.

The landmark matches are searched for using nearest- neighbor-distance ratio matching ${ }^{17)}$; there is a match between descriptor $d_{r 1}$ in the reference image and $d_{q}$ in the queue image if $\left|d_{r 2}-d_{q}\right| /\left|d_{r 1}-d_{q}\right|>t$, where $d_{r 1}$ is the first nearest neighbor and $d_{r 2}$ is the second nearest neighbor to $d_{q}$ and $t$ is the threshold, which we set to 1.5.

\section{Experimental Setup and Results}

\subsection{Scope of experiment}

We investigated the performances of the interest-region detectors and local descriptors using a series of images of Asteroid 25143 (Itokawa) taken by the Hayabusa spacecraft on 30 September and 1 October 2005. These images were taken sequentially from the home position, which was about $7 \mathrm{~km}$ from the asteroid. The asteroid rotated by about $1-3^{\circ}$ between every image and the spacecraft remained almost stationary; this can be regarded as the asteroid remained almost stationary and the spacecraft revolving.

In the actual mission, particularly for global mapping, it is desirable that landmarks be tracked not only in contiguous pairs of images, but also in multiple images to ensure accuracy and robustness. In this experiment, we calculated the performance scores for various angles of rotation from approximately 1 to $45^{\circ}$.

\subsection{Experimental setup}

For the region density, uniformity and repeatability, we investigated the SIFT (DoG), SURF, BRISK (AGAST), ORB (FAST), Harris-Affine, Hessian-Affine and MSER detectors. For precision and recall, we investigated SIFT, SURF, BRISK, ORB and combinations of Harris-Affine, HessianAffine and MSER detectors with the SIFT and ORB descriptors. Since the Harris-Affine and Hessian-Affine detectors generate elliptic regions, we normalized them to circles. ${ }^{26)}$ The elliptic regions were first resized to $31 \times 31$ patches by nearest-neighbor interpolation, then their dominant orientation was estimated using the technique employed in SIFT ${ }^{17)}$ and rotated accordingly before the descriptors were calculated. Note that MSER detects arbitrary regions, but they are first normalized to ellipses using intensity momentum.

The parameters of the detectors and descriptors were set to the default values of each implementation except for ORB, as stated in Section 3. In the evaluation of the repeatability, precision and recall, the number of ORB regions was limited to 1,000 since ORB generates more than 2,000 regions per image, which include many meaningless regions in the dark area of the image.

In the following, the detectors attached to certain descriptors (SIFT, BRISK and ORB) are referred to by the names of the descriptors, regardless of the original names of the detectors (i.e., DoG, AGAST and FAST) for clarity.

\subsection{Result 1: Region density and uniformity}

Table 2 shows the region density and uniformity scores. There is no major difference between the two categories except for ORB and MSER. The ORB detector extracted considerably more regions per image than the other detectors, which was followed by the SIFT detector. Note that all of the detectors except MSER extracted more than 70 regions, 
which was the number of landmarks configured in the Hayabusa mission. ${ }^{5)}$ The SIFT detector had the best uniformity score, although the scores of SURF, BRISK, ORB and Hessian-Affine were not greatly different from that of SIFT.

A density of 0.003 [regions/px] means that a region is detected every 18-square-pixel (about $12 \mathrm{~m}^{2}$ ) patch of the image on average. In the images of Itokawa taken from a distance of $7 \mathrm{~km}, 1$ pixel is about $0.7 \mathrm{~m}$; thus, a $12 \mathrm{~m}^{2}$ patch of the asteroid surface generates a single interest region.

\subsection{Result 2: Region repeatability}

Fig. 5 shows the repeatability scores of all the detectors plotted against the rotation of the asteroid, and Table 3 shows typical scores for smaller and larger rotations. Fig. 5

Table 2. Average region density and uniformity scores.

\begin{tabular}{llccc}
\hline Detector & $\begin{array}{c}\text { Density } \\
\text { [regions/px] }\end{array}$ & $\begin{array}{c}\text { \#Regions/ } \\
\text { image }\end{array}$ & Uniformity \\
\hline Cat.1 & SIFT & 0.0046 & 622 & $\mathbf{4 , 2 1 3}$ \\
& SURF & 0.0017 & 227 & 4,104 \\
& BRISK & 0.0018 & 240 & 4,062 \\
& ORB & $\mathbf{0 . 0 2 0}$ & $\mathbf{2 , 6 5 4}$ & 4,210 \\
\hline Cat.2 & Harris-Affine & 0.0011 & 139 & 3,355 \\
& Hessian-Affine & 0.0030 & 395 & 4,011 \\
& MSER & 0.00033 & 43 & 2,979 \\
\hline
\end{tabular}

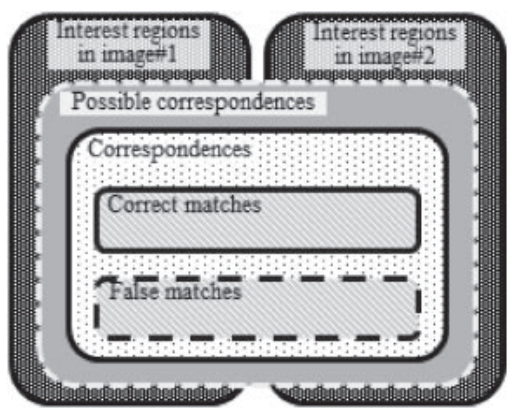

(a)

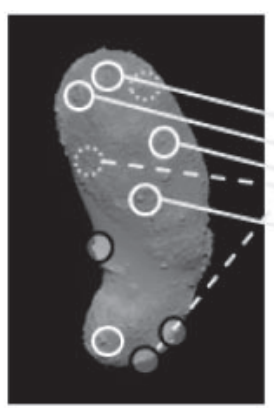

(b) indicates that techniques in Category 1 performed well, particularly for the smaller angle of rotation, and techniques in Category 2 generally failed. In each category, repeatability scores were similar to each other. Note that Harris-Affine performed slightly better for the larger angle of rotation.

The moderate performances of the affine-invariant detectors may partly stem from the fact that they generate elliptical regions. The probability that two elliptical regions are regarded as corresponding may be lower than that of the circular regions because the noise in the orientation estimation can affect the repeatability of detected regions.

Table 3. Repeatability scores for smaller and larger angles.

\begin{tabular}{llcc}
\hline \multirow{2}{*}{ Cat.1 } & Detector & \multicolumn{2}{c}{ Repeatability } \\
\cline { 3 - 4 } & SIFT & $\mathbf{0 . 6 9}$ & $\theta=32^{\circ}$ \\
& SURF & 0.54 & 0.21 \\
& BRISK & 0.55 & 0.20 \\
& ORB & 0.60 & 0.17 \\
\hline \multirow{2}{*}{ Cat.2 } & Harris-Affine & 0.30 & 0.18 \\
& Hessian-Affine & 0.34 & $\mathbf{0 . 2 8}$ \\
& MSER & 0.30 & 0.20 \\
\hline
\end{tabular}
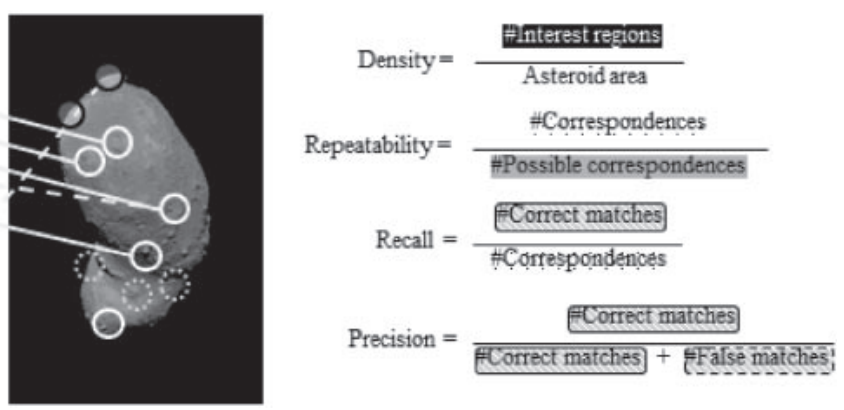

(c)

Fig. 4. (a) Comprehensive diagram of region and descriptor relations, (b) their examples and (c) criteria used in this paper. In (b), the white solid circles are the correspondences that are detected in corresponding sites, the dotted circles are the possible correspondences that could be detected in another image and the black solid circles are the regions that are not visible in another image. The solid lines denote the correct matches and the dotted ones denote the false matches.

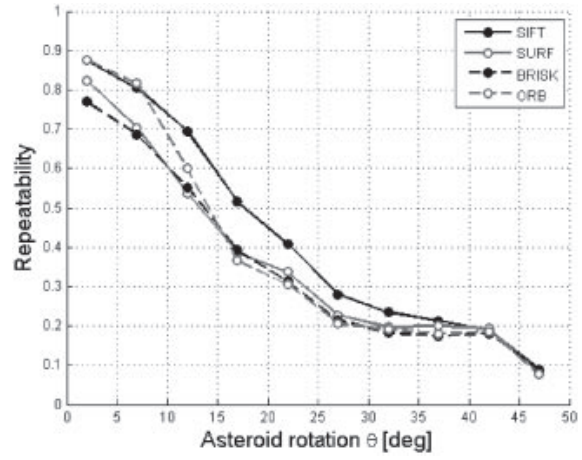

(a)

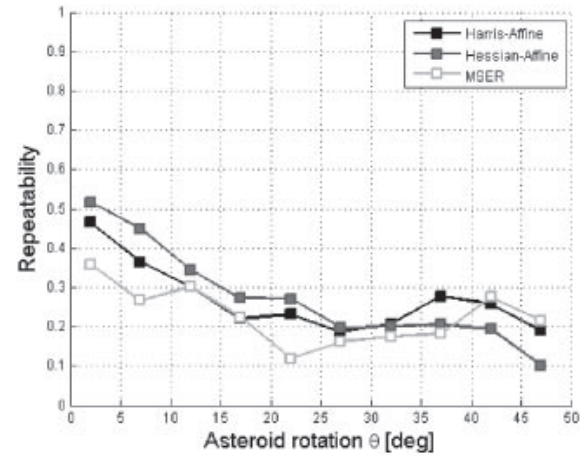

(b)

Fig. 5. (a) Repeatability scores of scale- and rotation-invariant detectors (Category 1), and (b) repeatability scores of affine-invariant detectors (Category 2).

A larger repeatability score leads to better tracking. 


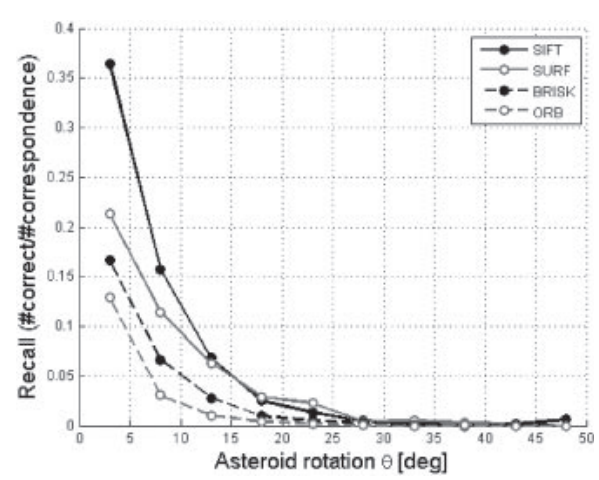

(a)

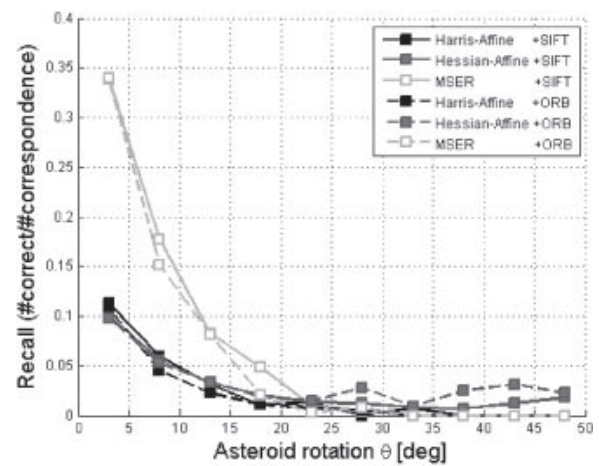

(b)

Fig. 6. (a) Recall scores of scale- and rotation-invariant detectors (Category 1), and (b) precision scores of affine-invariant detectors (Category 2).

A larger recall score leads to more robust navigation.

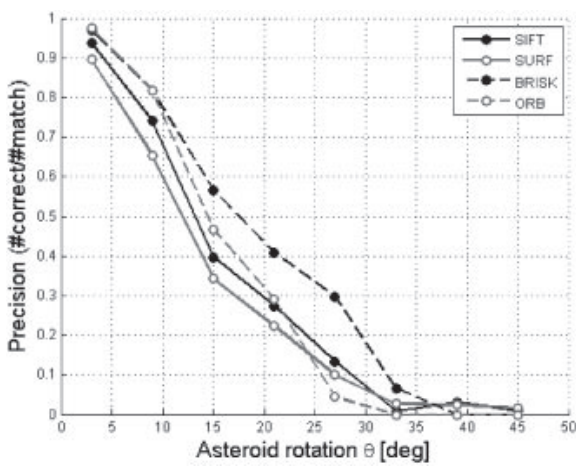

(a)

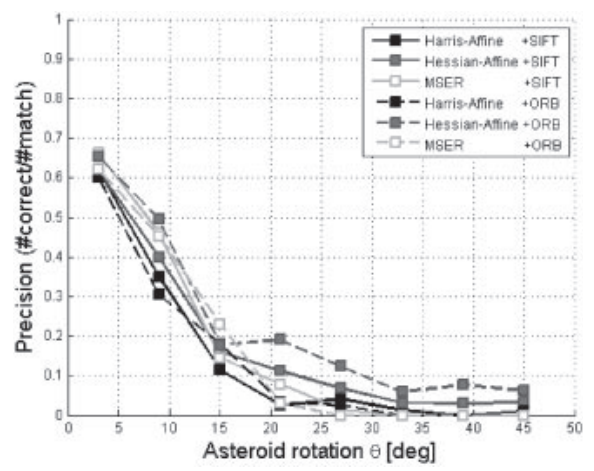

(b)

Fig. 7. (a) Precision scores of scale- and rotation-invariant detectors (Category 1), and (b) precision scores of affine-invariant detectors (Category 2).

A larger precision score leads to more accurate navigation.

Table 4. Recall and precision scores for smaller and larger angles.

\begin{tabular}{lllllll}
\hline & \multirow{2}{*}{ Detector + Descriptor } & \multicolumn{2}{c}{ Recall } & & \multicolumn{2}{c}{ Precision } \\
\cline { 3 - 4 } \cline { 6 - 7 } & & $\theta=8^{\circ}$ & $\theta=28^{\circ}$ & & $\theta=15^{\circ}$ & $\theta=38^{\circ}$ \\
\hline Cat.1 & SIFT & 0.15 & 0.005 & & 0.40 & 0.032 \\
& SURF & 0.11 & 0.004 & & 0.34 & 0.024 \\
& BRISK & 0.066 & 0.003 & & $\mathbf{0 . 5 9}$ & 0 \\
& ORB & 0.030 & 0 & 0.49 & 0 \\
\hline Cat.2 & Harris-Affine + SIFT & 0.059 & 0 & & 0.11 & 0 \\
& Hessian-Affine + SIFT & 0.056 & 0.012 & & 0.16 & 0.032 \\
& MSER + SIFT & $\mathbf{0 . 1 8}$ & 0.004 & & 0.15 & 0 \\
& Harris-Affine + ORB & 0.045 & 0.006 & & 0.18 & 0 \\
& Hessian-Affine + ORB & 0.053 & $\mathbf{0 . 0 2 8}$ & & 0.18 & $\mathbf{0 . 0 7 7}$ \\
& MSER + ORB & 0.15 & 0.008 & 0.23 & 0 \\
\hline
\end{tabular}

\subsection{Result 3: Matching recall}

Fig. 6 shows the recall scores and Table 4 shows typical scores for smaller and larger rotations. The performances of Category 1 except SIFT and Category 2 except MSERs were similarly moderate. SIFT and MSERs were significantly better recalling than other techniques. Note that with increasing rotation, Hessian-Affine+ORB slightly outperformed the other combinations.

\subsection{Result 4: Matching precision}

Fig. 7 shows the precision scores and Table 4 shows typical scores for smaller and larger rotations. The scale- and rotation-invariant techniques (Category 1) had better precision than the affine-invariant techniques' scores (Category 2) when the rotation of the asteroid was less than $30^{\circ}$. In particular, BRISK showed the best recall of Category 1. The precision scores of Category 2 were slightly better when the asteroid rotation was large.

Examples of the differences in precision scores are shown in Fig. 8 for clarity, where solid lines show correct matches and dotted lines show false matches. The low precision scores mean that careful elimination of the false matches is required, but it is difficult to eliminate them automatically in nonplanar scenes such as asteroid images.

The moderate performances of combinations of affine-invariant detectors and SIFT or ORB descriptors may stem from the region normalization process, whose interpolation method can affect the output of local descriptors. The performances of the affine-invariant detectors may improve with further consideration of the normalization process.

\section{Discussion: Which Detector/Descriptor to Use?}

On the basis of the evaluation results, we provide some suggestions as to which method should be used. Generally, the scale- and rotation-invariant techniques (Category 1) per- 


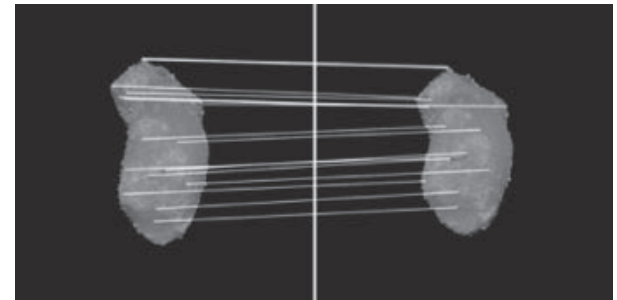

(a) BRISK

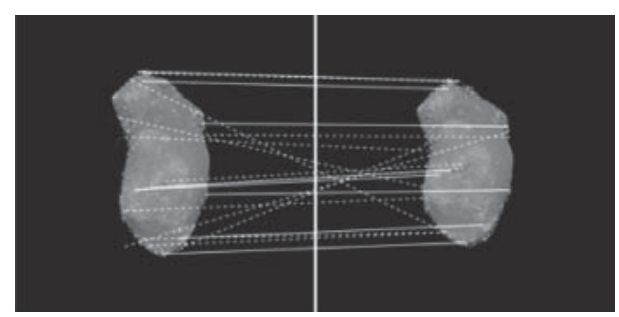

(b) Hessian-Affine+ORB

Fig. 8. Examples of matches with different precision scores: (a) matches of BRISK and (b) matches of Hessian-Affine + ORB.

The solid lines denote correct matches and dotted lines denote false matches. Note that (a) contains no false matches but (b) contains many false matches.

Table 5. Performance summary of the detectors and descriptors.

\begin{tabular}{llcccc}
\hline & Method & $\begin{array}{c}\text { Density \& } \\
\text { Uniformity }\end{array}$ & Repeatability & Recall & Precision \\
\hline Cat.1 & SIFT & Good & Good & Good & Good \\
& SURF & Good & Good & Good & Fair \\
& BRISK & Good & Good & Fair & Good \\
& ORB & Good & Good & Fair & Good \\
\hline Cat.2 & Harris-Affine & Moderate & Fair & Fair & Poor \\
& Hessian-Affine & Good & Fair & Fair & Poor \\
& MSER & Poor & Moderate & Good & Poor \\
\hline
\end{tabular}

formed better than the affine-invariant techniques (Category 2) (see Table 5).

With regard to the region density and uniformity (Table 2), MSER generates too few interest regions to be used. This could be improved by tuning the parameters, but there is no particular motivation to do this for MSER as the other affine-invariant detectors, Harris-Affine and Hessian-Affine, generate a sufficient number of regions. This tendency of MSER to generate a small number of regions was also observed in a previous study. ${ }^{28)}$

The region repeatability scores (Fig. 5) have a moderate amount of importance in the selection of which detector to use, because the noncorresponding regions can be rejected provided that a sufficient number of interest regions are detected. The experimental results indicate that the affine-invariant detectors outperform other detectors for a large angle of asteroid rotation.

SIFT was the best at recalling, followed by MSER and SURF (Fig. 6). Note that all of the detectors and descriptors have difficulty recalling corresponding interest regions when the angle of asteroid rotation is more than $20^{\circ}$. This suggests we should design a landmark tracking system that searches for region matches among images that are as close to each other as possible.

The matching precision scores (Fig. 7) were very high when the angle of asteroid rotation was less than approximately $10^{\circ}$. In particular, SIFT, BRISK and ORB showed good precision scores. For a larger angle of rotation, the precision scores were almost zero generally, which means that descriptor matching is ineffective between images of the asteroid with a large rotation angle. Accordingly, we recommend a landmark tracking system based on matching between close images.

For all of the above reasons, SIFT can be used for automatic global mapping and visual navigation with slight tuning of parameters, and SURF, BRISK and ORB will be acceptable with careful tuning of the parameters. On the other hand, although the affine-invariant detectors may exhibit superior feature tracking, their use is more challenging owing to the extra normalization process for elliptical or arbitrary-shaped regions.

Note that since our experiments use the images and the model of Itokawa, their results may not be applicable to every celestial body in entirely the same way. However, the photometric conditions for Itokawa's case are not special in space; hence, our performance evaluation can be referred to when treating other asteroids with standard conditions.

When it is necessary to evaluate performances for other celestial bodies in particular, one can adopt the evaluation procedures presented in this paper. Our procedures can be used not only for asteroids, but also for other general bodies including terrestrial terrain if geometric models are available. The resolution of the models should be as high as about 10, or more vertices should be included in the smallest interest regions, from our experiences.

\section{Conclusion}

In this study, we evaluated the performances of several interest-region detectors and local descriptors of asteroid images for automatic global mapping and visual navigation. As a result, we found that SIFT is acceptable for use, while SURF, BRISK and ORB can be used with careful tuning of the parameters. The affine-invariant detectors might contribute to more accurate feature tracking, but utilizing them requires further research of an appropriate region-normalizing process.

In future research, we will design an asteroid-specific descriptor so that dramatic changes in appearance due to the lack of scattering light and nonplanarity can be captured. We also plan to merge automatic feature tracking with the subsequent global mapping and visual navigation based on SFM or SLAM and evaluate overall performance.

\section{References}

1) Yano, H., Kubota, T., Miyamoto, H., Okada, T., Scheeres, D., Takagi, Y., Yoshida, K., Abe, M., Abe, S., Barnouin-Jha, O., Fujiwara, A., Hasegawa, S., Hashimoto, T., Ishiguro, M., Kato, M., Kawaguchi, J., Mukai, T., Saito, T., Sasaki, S., and Yoshikawa, M.: Touchdown 
of the Hayabusa Spacecraft at the Muses Sea on Itokawa, Science, 312 (2006), pp. 1350-1353.

2) Maruya, M., Ohyama, H., Uo, M., Muranaka, N., Morita, H., Kubota, T., Hashimoto, T., Saito, J., and Kawaguchi, J.: Navigation Shape and Surface Topography Model of Itokawa, AIAA Guidance, Navigation, and Control Conf., 2006.

3) Demura, H., Kobayashi, S., Nemoto, E., Matsumoto, N., Furuya, M., Yukishita, A., Muranaka, N., Morita, H., Shirakawa, K., Maruya, M., Ohyama, H., Uo, M., Kubota, T., Hashimoto, T., Kawaguchi, J., Fujiwara, A., Saito, J., Sasaki, S., Miyamoto, H., and Hirata, N.: Pole and Global Shape of 25143 Itokawa, Science, 312 (2006), pp. 1347-1349.

4) Gaskell, R., Barnouin-Jha, O., Scheeres, D., Mukai, T., Hirata, N., Abe, S., Saito, J., Ishiguro, M., Kubota, T., Hashimoto, T., Kawaguchi, J., Yoshikawa, M., Shirakawa, K., and Kominato, T.: Landmark Navigation Studies and Target Characterization in the Hayabusa Encounter with Itokawa, AIAA/AAS Astrodynamics Specialist Conf. and Exhibit, 2006.

5) Shirakawa, K., Morita, H., Uo, M., Hashimoto, T., Kubota, T., and Kawaguchi, J.: Accurate Landmark Tracking for Navigating Hayabusa Prior to Final Descent, Adv. Astronaut. Sci., 124 (2006), pp. 1817-1826.

6) Yoshikawa, M. and Hayabusa-2 Project Team: Outline of the Next Asteroid Sample Return Mission of Japan-Hayabusa-2, 28th Int. Symposium on Space Technol. and Sci., 2011.

7) Szeliski, R.: Computer Vision: Algorithms and Applications, Springer, Berlin, 2010.

8) Tomasi, C. and Kanade, T.: Shape and Motion from Image Streams under Orthography: A Factorization Method, Int. J. Comput. Vision, 9 (1992), pp. 137-154.

9) Triggs, B., McLauchlan, P. F., Hartley, R. I., and Fitzgibbon, A. W.: Bundle Adjustment-A Modern Synthesis, Vision Algorithms: Theory and Practice, Springer, Berlin, 2000, pp. 298-372.

10) Thrun, S., Burgard, W., and Fox, D.: Probabilistic Robotics, The MIT Press, Cambridge, 2012.

11) Cocaud, C. and Kubota, T.: Monocular SLAM-Based Navigation for Autonomous Approach Descent and Landing on Small Celestial Bodies, Int. Symposium on Artificial Intelligence, Robotics and Automation in Space, 2012.

12) Mori, Y., Hirata, N., Demura, H., Naruse, K., and Yaguchi, Y.: 3D Reconstruction of Asteroid using Structure from Motion, 29th Int. Symposium on Space Technol. and Sci., 2013.

13) Snavely, N., Seitz, S. M., and Szeliski, R.: Photo Tourism: Exploring Photo Collections in 3D, ACM Trans. Graphics, 25 (2006), pp. 835-846.

14) Tanimoto, A., Takeishi, N., Yairi, T., Tsuda, Y., Terui, F., Ogawa, N., and Mimasu, Y.: Simultaneous Estimation of Asteroid Shape and Motion for Spacecraft Navigation, Robotics and Biomimetics, IEEE
Int. Conference, 2013

15) Yairi, T.: Co-embedding of Structurally Missing Data by Locally Linear Alignment, Lecture Notes in Comput. Sci., 7302 (2012), pp. 419-430.

16) Takeishi, N.: Automatic Landmark Recognition for Asteroid by Image Features, 29th Int. Symposium on Space Technol. and Sci., 2013.

17) Lowe, D. G.: Distinctive Image Features from Scale-Invariant Keypoints, Int. J. Comput. Vision, 60 (2004), pp. 91-110.

18) Bay, H., Ess, A., Tuytelaars, T., and Gool, L. V.: SURF: Speeded Up Robust Features, Comput. Vision Image Understanding, 110 (2008), pp. 346-359.

19) Calonder, M., Lepetit, V., Strecha, C., and Fua, P.: BRIEF: Binary Robust Independent Elementary Features, Lecture Notes in Comput. Sci., 6314 (2010), pp. 778-792.

20) Rosten, E. and Drummond, T.: Machine Learning for High-speed Corner Detection, Lecture Notes in Comput. Sci., 3952 (2006), pp. $430-443$.

21) Leutenegger, S., Chli, M., and Siegwart, R. Y.: BRISK: Binary Robust invariant scalable keypoints, Proc. of Comput. Vision, IEEE Int. Conf., 2011, pp. 2548-2555.

22) Mair, E., Hager, G. D., Burschka, D., Suppa, M., and Hirzinger, G.: Adaptive and Generic Corner Detection Based on the Accelerated Segment Test, Lecture Notes in Comput. Sci., 6314 (2010), pp. 183-196.

23) Rublee, E., Rabaud, V., Konolige, K., and Bradski, G.: ORB: An Efficient Alternative to SIFT or SURF, Proc. of Comput. Vision, IEEE Int. Conf., 2011, pp. 2564-2571.

24) Mikolajczyk, K. and Schmid, C.: An Affine Invariant Interest Point Detector, Lecture Notes in Comput. Sci., 2350 (2002), pp. 128-142.

25) Matas, J., Chum, O., Urban, M., and Pajdla, T.: Robust Wide-baseline Stereo from Maximally Stable Extremal Regions, Proc. of British Machine Vision Conf., 2002, pp. 384-396.

26) Mikolajczyk, K., Tuytelaars, T., Schmid, C., Zisserman, A., Matas, J., Schaffalitzky, F., Kadir, T., and Gool, L. V.: A Comparison of Affine Region Detectors, Int. J. Comput. Vision, 65, 2-1 (2005), pp. 43-72.

27) Mikolajczyk, K. and Schmid, C.: A Performance Evaluation of Local Descriptors, Pattern Anal. Machine Intelligence IEEE Trans., 27, 10 (2005), pp. 1615-1630.

28) Fraundorfer, F. and Bischof, H.: A Novel Performance Evaluation Method of Local Detectors on Non-planar Scenes, Comput. Vision and Pattern Recognition-Workshops, IEEE Comput. Soc. Conf., 2005.

29) Heinly, J., Dunn, E., and Frahm, J. M.: Comparative Evaluation of Binary Features, Lecture Notes in Comput. Sci., 7572 (2012), pp. 759-773.

30) Harris, C. and Stephens, M.: A Combined Corner and Edge Detector, Alvey Vision Conf., 15 (1988), p. 50.

31) Tuytelaars, T. and Van Gool, L.: Matching Widely Separated Views Based on Affine Invariant Regions, Int. J. Comput. Vision, 59 (2004), pp. 61-85. 